\title{
APPROXIMATING FIXED POINTS OF NONEXPANSIVE MAPPINGS
}

\author{
H. F. SENTER AND W. G. DOTSON, JR.
}

\begin{abstract}
A condition is given for nonexpansive mappings which assures convergence of certain iterates to a fixed point of the mapping in a uniformly convex Banach space. A relationship between the given condition and the requirement of demicompactness is established.
\end{abstract}

Introduction. Browder [1] and Kirk [7] have shown that a nonexpansive mapping $T$ which maps a closed, bounded, convex subset $C$ of a uniformly convex Banach space into itself has a nonempty fixed point set in $C$. In general, however, for arbitrary $x \in C$ the Picard iterates $T^{n} x$ do not converge to a fixed point of $T$. It will be shown that if $T$ satisfies one additional condition, then an iterative process of the type introduced by W. R. Mann [8] converges to a fixed point of $T$. For nonexpansive mappings $T$ which have fixed points, this additional condition is weaker than the requirement that $T$ be demicompact.

Convergence to a fixed point. Let $X$ be a Banach space with norm $|\cdot|$ and $C$ a convex subset of $X$. A self-mapping $T$ of $C$ is said to be nonexpansive provided $|T x-T y| \leqq|x-y|$ holds for all $x, y \in C$. A mapping $T: C \rightarrow C$ with nonempty fixed point set $F$ in $C$ will be said to satisfy Condition $\mathrm{I}$ if there is a nondecreasing function $f:[0, \infty) \rightarrow[0, \infty)$ with $f(0)=0, f(r)>0$ for $r \in(0, \infty)$, such that $|x-T x| \geqq f(d(x, F))$ for all $x \in C$, where $d(x, F)=\inf \{|x-z|: z \in F\}$.

Let $P$ denote the set of positive integers. For $x_{1} \in C, M\left(x_{1}, t_{n}, T\right)$ is the sequence $\left\{x_{n}\right\}$ defined by $x_{n+1}=\left(1-t_{n}\right) x_{n}+t_{n} T x_{n}$ where $t_{n} \in[a, b]$ for all $n \in P$ and $0<a<b<1$. This iterative process has been previously investigated by Dotson in [4].

Our main result for nonexpansive mappings is the following:

THeOREm 1. Suppose $X$ is a uniformly convex Banach space, $C$ is a closed, bounded, convex, nonempty subset of $X$, and $T$ is a nonexpansive mapping of $C$ into $C$. Let $F$ denote the fixed point set of $T$ in $C$, and suppose

Received by the editors February 26, 1973.

AMS (MOS) subject classifications (1970). Primary 47H10.

Key words and phrases. Iteration, nonexpansive mappings, fixed point theorems, demicompact mappings.

(c) American Mathematical Society 1974 
$T$ satisfies Condition I. Then for any $x_{1} \in C, M\left(x_{1}, t_{n}, T\right)$ converges to a member of $F$.

Given that $F$ is nonempty (which in Theorem 1 is assured by the BrowderKirk theorem [1], [7]) the proof that $M\left(x_{1}, t_{n}, T\right)$ converges to a fixed point uses only the fact that $T$ is nonexpansive about its fixed points (see Theorem 2 below). Theorem 1 will follow immediately as a corollary of Theorem 2. As in [3], a self-mapping $T$ of $C$ will be called quasinonexpansive provided $T$ has a fixed point in $C$ and if $p \in C$ is a fixed point of $T$ then $|T x-p| \leqq|x-p|$ is true for all $x \in C$. The class of quasinonexpansive mappings includes continuous as well as discontinuous mappings which are not nonexpansive. One can easily prove that $T: C \rightarrow C$ is quasi-nonexpansive if $T$ has a fixed point in $C$ and for $x, y \in C$ satisfies either

$$
|T x-T y| \leqq \beta[|x-T x|+|y-T y|], \quad 0 \leqq \beta \leqq 1 / 2,
$$

or

$$
|T x-T y| \leqq a|x-T x|+b|y-T y|+c|\dot{x}-y|,
$$

where $a, b, c>0$ and $a+b+c \leqq 1$.

Mappings which satisfy the requirement (A) or (B) have been recently investigated by Kannan [6] and Reich [12] respectively.

For a uniformly convex Banach space $X$, Dotson [4] has shown that if $\left\{w_{n}\right\}$ and $\left\{y_{n}\right\}$ are sequences in the closed unit ball of $X$ and if $\left\{z_{n}\right\}=$ $\left\{\left(1-t_{n}\right) w_{n}+t_{n} y_{n}\right\}$ satisfies $\lim \left|z_{n}\right|=1$, where $t_{n} \in[a, b]$ for $0<a<b<1$, then $\lim \left|w_{n}-y_{n}\right|=0$. This result will be used in the proof of

THEOREM 2. Suppose $X$ is a uniformly convex Banach space, $C$ is a closed, convex subset of $X$ and $T$ is a quasi-nonexpansive mapping of $C$ into $C$. If $T$ satisfies Condition I, where $F$ is the fixed point set of $T$ in $C$, then for arbitrary $x_{1} \in C, M\left(x_{1}, t_{n}, T\right)$ converges to a member of $F$.

Proof. If $x_{1} \in F$ the result is trivial, so we assume $x_{1} \in C \sim F$. For arbitrary $z \in F$ we have for $n \in P$ that $\left|T x_{n}-z\right| \leqq\left|x_{n}-z\right|$ and so

$$
\left|x_{n+1}-z\right| \leqq\left(1-t_{n}\right)\left|x_{n}-z\right|+t_{n}\left|T x_{n}-z\right| \leqq\left|x_{n}-z\right| \text {. }
$$

Thus, $d\left(x_{n+1}, F\right) \leqq d\left(x_{n}, F\right)$ for all $n \in P$. The sequence $\left\{d\left(x_{n}, F\right)\right\}$ is nonincreasing and bounded below, so $\lim d\left(x_{n}, F\right)$ exists. We now show (indirectly) that this limit must be zero, and in turn, that $\left\{x_{n}\right\}$ converges to a member of $F$.

Suppose $\lim d\left(x_{n}, F\right)=b>0$. Then for $z_{0} \in F, \lim \left|x_{n}-z_{0}\right|=b^{\prime} \geqq b>0$. Choose $N>0$ such that $\left|x_{n}-z_{0}\right| \leqq 2 b^{\prime}$ for $n \geqq N$. If we let $y_{n}=$ $\left(T x_{n}-z_{0}\right) /\left|x_{n}-z_{0}\right|$ and $w_{n}=\left(x_{n}-z_{0}\right) /\left|x_{n}-z_{0}\right|$, then $\left|y_{n}\right| \leqq 1$ and $\left|w_{n}\right|=1$ 
for all $n \in P$; and for $n \geqq N$

$$
\left|w_{n}-y_{n}\right|=\frac{\left|x_{n}-T x_{n}\right|}{\left|x_{n}-z_{0}\right|} \geqq \frac{f\left(d\left(x_{n}, F\right)\right)}{\left|x_{n}-z_{0}\right|} \geqq \frac{f(b)}{2 b^{\prime}}>0 .
$$

Therefore, $\lim \left|w_{n}-y_{n}\right| \neq 0$. Moreover

$$
\lim \left|\left(1-t_{n}\right) w_{n}+t_{n} y_{n}\right|=\lim \left|x_{n+1}-z_{0}\right| /\left|x_{n}-z_{0}\right|=b^{\prime} \mid b^{\prime}=1 .
$$

However, by the contrapositive of Dotson's result [4] stated above, since $\lim \left|w_{n}-y_{n}\right| \neq 0$ then the existence of $\lim \left|\left(1-t_{n}\right) w_{n}+t_{n} y_{n}\right|$ implies $\lim \left|\left(1-t_{n}\right) w_{n}+t_{n} y_{n}\right| \neq 1$, a contradiction. Therefore, $\lim d\left(x_{n}, F\right)=0$. We show that this implies $\left\{x_{n}\right\}$ converges to an element of $F$.

Since $\lim d\left(x_{n}, F\right)=0$, given $\varepsilon>0$ there exists $N_{\varepsilon}>0$ and $z_{\varepsilon} \in F$ such that $\left|x_{N_{\varepsilon}}-z_{\varepsilon}\right|<\varepsilon$, which implies $\left|x_{n}-z_{\varepsilon}\right|<\varepsilon$ for all $n \geqq N_{\varepsilon}$. Thus, if $\varepsilon_{k}=1 / 2^{k}$ for $k \in P$, then corresponding to each $\varepsilon_{k}$ there is an $N_{k}>0$ and a $z_{k} \in F$ such that $\left|x_{n}-z_{k}\right| \leqq \varepsilon_{k} \mid 4$ for all $n \geqq N_{k}$. We require $N_{k+1} \geqq N_{k}$ for all $k \in P$. We have for all $k \in P$

$$
\left|z_{k}-z_{k+1}\right|=\left|z_{k}-x_{N_{k+1}}+x_{N_{k+1}}-z_{k+1}\right|<\varepsilon_{k} / 4+\varepsilon_{k+1} / 4=3 \varepsilon_{k+1} / 4 .
$$

Let $S(z, \varepsilon)=\{x \in X:|x-z| \leqq \varepsilon\}$ denote the closed sphere centered at $z$ of radius $\varepsilon$. For $x \in S\left(z_{k+1}, \varepsilon_{k+1}\right)$ we have

$$
\left|z_{k}-x\right|=\left|z_{k}-z_{k+1}+z_{k+1}-x\right|<3 \varepsilon_{k+1} / 4+\varepsilon_{k+1}<2 \varepsilon_{k+1}=\varepsilon_{k} .
$$

That is, $S\left(z_{k+1}, \varepsilon_{k+1}\right) \subseteq S\left(z_{k}, \varepsilon_{k}\right)$ for $k \in P$. Thus, $\left\{S\left(z_{k}, \varepsilon_{k}\right)\right\}$ is a nested sequence of nonvoid closed spheres with radii $\varepsilon_{k}$ tending to zero. By the Cantor intersection theorem, $\bigcap_{k \in P} S\left(z_{k}, \varepsilon_{k}\right)$ contains exactly one point, say $w$. The fixed point set $F$ is closed by [3] and the sequence $\left\{z_{k}\right\}$ from $F$ converges to $w$, so $w \in F$. Since $\left|x_{n}-z_{k}\right|<\varepsilon_{k} \mid 4$ for $n \geqq N_{k}$, we have $\left\{x_{n}\right\} \rightarrow w$. Q.E.D.

Note that in Theorem 2 the set $C$ is not required to be bounded; however, boundedness of $C$ is needed in Theorem 1 to apply the BrowderKirk theorem.

In the preceding theorems, the fixed point of $T$ to which $M\left(x_{1}, t_{n}, T\right)$ converges depends, in general, on the initial approximation $x_{1}$ as well as the values of the $t_{n}$. Also, $M\left(x_{1}, t_{n}, T\right)$ need not converge to the fixed point of $T$ nearest $x_{1}$. The following example can be used to verify each of these facts. Let $X$ be the space $R^{2}$ with the Euclidean norm and, with $(r, \theta)$ denoting polar coordinates, let $C=\{(r, \theta): 0 \leqq r \leqq 1,-\pi / 2 \leqq \theta \leqq$ $-\pi / 4\}$. Define $T: C \rightarrow C$ by $T[(r, \theta)]=(r,-\pi / 2)$ for each point $(r, \theta)$ in $C$. The set of fixed points of $T$ is the line segment $F=\{(r,-\pi / 2): 0 \leqq r \leqq 1\}$. 
On Condition I. If $T: C \rightarrow C$ has a nonvoid fixed point set $F$, then $T$ will be said to satisfy Condition II provided there exists a real number $\alpha>0$ such that $|x-T x| \geqq \alpha \cdot d(x, F)$ holds for all $x \in C$, where as before $d(x, F)=\inf _{z \in F}|x-z|$. Clearly mappings which satisfy Condition II also satisfy Condition I, and in some cases Condition II is easily verified. In the example above, Condition II holds with $\alpha=1$. If $T$ rotates points of the unit ball of $R^{2}$ through an angle $\pi / 2$, then Condition II holds with $\alpha=\sqrt{ } 2$.

Condition II is similar to, but less restrictive than, a requirement imposed by Outlaw in [10, Theorem 2]. Mappings satisfying Outlaw's condition can have at most a single fixed point; his second theorem follows as a special case of Theorem 2 .

If $T: C \rightarrow C$ satisfies either requirement (A) or (B) (see above) and has a fixed point in $C$, then it is easily shown that $T$ has a unique fixed point [6], [12]. In [6, Theorem 2] Kannan proves under certain conditions that for $x_{1} \in C, M\left(x_{1}, \frac{1}{2}, T\right)$ converges to the fixed point of $T$ if $T$ satisfies (A). We extend his result with

THEOREM 3. Let $C$ be a subset of a Banach space $X$ and $T$ a mapping of $C$ into $C$ which satisfies either (A) or (B) and has a (unique) fixed point in $C$. Then $T$ satisfies Condition II. If $C$ is closed and convex and $X$ is uniformly convex then for any $x_{1} \in C, M\left(x_{1}, t_{n}, T\right)$ converges to the fixed point of $T$.

Proof. Assume $T$ satisfies requirement (B) and let $p$ be the unique fixed point of $T$. Then for $x \in C$

and

$$
|T x-p|=|T x-T p| \leqq a|x-T x|+c|x-p|
$$

$$
|T x-p| \geqq|| T x-x|-| x-p|| \geqq|x-p|-|x-T x| .
$$

Hence

$$
a|x-T x|+c|x-p| \geqq|x-p|-|x-T x|,
$$

so $|x-T x| \geqq[(1-c) /(1+a)]|x-p|$. The constant $(1-c) /(1+a)$ is positive since $0<a, c<1$. Thus Condition II holds. A similar argument applies if $T$ is a mapping of the type (A).

Since $T$ is quasi-nonexpansive and satisfies Condition $I$, the second assertion of the theorem follows directly from Theorem 2 . Q.E.D.

We now establish a relationship between mappings which satisfy Condition I and those which are demicompact, beginning with

LEMma 1. Suppose $C$ is a closed, bounded subset of a Banach space $X$ and $T: C \rightarrow C$ has a nonempty fixed point set $F$ in $C$. If $I-T$ maps closed bounded subsets of $C$ onto closed subsets of $X$, then $T$ satisfies Condition I on $C$. 
Proof. Let $M=\sup \{d(x, F): x \in C\}$. If $M=0$ then $F=C$ and Condition I follows trivially. Suppose $M>0$; for $0<r<M$ define $C_{r}=\{x \in C$ : $d(x, F) \geqq r\}$ and $f(r)=\inf \left\{|x-T x|: x \in C_{r}\right\}$. Note that each set $C_{r}$ is nonempty, closed and bounded. We prove that for arbitrary $r, 0<r<M$, $f(r)>0$.

By hypothesis, $(I-T) C_{r}=\left\{x-T x: x \in C_{r}\right\}$ is closed. If $\theta \in(I-T) C_{r}$ then $\theta=z-T z$ for some $z \in C_{r}$, which implies $z=T z$ and thus $z \in F$. But $d(z, F) \geqq r>0$, a contradiction. Therefore, $\theta \notin(I-T) C_{r}$. Suppose now that $f(r)=0$. Then there is a sequence $\left\{x_{n}\right\} \subseteq C_{r}$ such that $\left|x_{n}-T x_{n}\right| \rightarrow 0$ and hence $\left\{x_{n}-T x_{n}\right\} \rightarrow \theta$. But $\left\{x_{n}-T x_{n}\right\} \subseteq(I-T) C_{r}$, a closed set. Thus we obtain $\theta \in(I-T) C_{r}$, contradicting our statement above that $\theta \notin(I-T) C_{r}$. Therefore, $f(r)>0$ for $0<r<M$.

We extend the domain of $f$ to $[0, \infty)$ by defining $f(0)=0$ and $f(r)=$ $\sup \{f(s): s<M\}$ for $r \geqq M$. It is easy to verify that $f$ so defined fulfills the hypotheses of Condition $\mathrm{I}$; in particular, $|x-T x| \geqq f(d(x, F))$ for each $x \in C$. Q.E.D.

A consequence of Lemma 1 and Theorem 2 is

Corollary 1 (Browder and Petryshyn [2]). Let $C$ be a closed, convex subset of a uniformly convex Banach space $X$ and $T: C \rightarrow C$ a nonexpansive mapping. For $\lambda \in(0,1)$ let $T_{\lambda}$ be given by $T_{\lambda}=\lambda I+(1-\lambda) T$. (Notice that $M\left(x_{1}, 1-\lambda, T\right)=\left\{T_{\lambda}^{n} x_{1}\right\}$.) If I-T maps closed bounded subsets of $C$ onto closed subsets of $X$ and if the set $F$ of fixed points of $T$ is nonempty, then for any $\lambda \in(0,1)$ and every $x$ in $C$ the sequence $\left\{T_{\lambda}^{n} x\right\}$ converges to a member of $F$.

A mapping $T: C \rightarrow X$ of a subset $C$ of a Banach space $X$ is said to be demicompact [11] provided whenever $\left\{x_{n}\right\} \subseteq C$ is bounded and $\left\{x_{n}-T x_{n}\right\}$ converges then there is a subsequence $\left\{x_{n_{i}}\right\}$ which converges. If a mapping $T$ is continuous as well as demicompact then, according to Opial [9, p. 41], the mapping $I-T$ maps closed bounded subsets of $C$ onto closed subsets of $X$. In particular, if $T: C \rightarrow C$ is nonexpansive and demicompact and has a fixed point in $C$, it follows from Opial's result and Lemma 1 that $T$ must satisfy Condition I. Using a different approach, Groetsch [5] has established the convergence of mean-value iterates of nonexpansive, demicompact mappings to a fixed point of the mapping.

\section{REFERENCES}

1. F. E. Browder, Nonexpansive nonlinear operators in a Banach space, Proc. Nat. Acad. Sci. U.S.A. 54 (1965), 1041-1044. MR 32 \#4574.

2. F. E. Browder and W. V. Petryshyn, The solution by iteration of nonlinear functional equations in Banach spaces, Bull. Amer. Math. Soc. 72 (1966), 571-575. MR 32 \#8155b. 
3. W. G. Dotson, Jr., Fixed points of quasi-nonexpansive mappings, J. Austral. Math. Soc. 13 (1972), 167-170. MR 45 \#7551.

4. - On the Mann iterative process, Trans. Amer. Math. Soc. 149 (1970), 65-73. MR 41 \#2477.

5. G. W. Groetsch, A note on segmenting Mann iterates, J. Math. Anal. Appl. 40 (1972), 369-372.

6. R. Kannan, Some results on fixed points. III, Fund. Math. 70 (1971), 169177. MR $44 \# 879$.

7. W. A. Kirk, A fixed point theorem for mappings which do not increase distances, Amer. Math. Monthly 72 (1965), 1004-1006. MR 32 \#6436.

8. W. R. Mann, Mean value methods in iteration, Proc. Amer. Math. Soc. 4 (1953), 506-510. MR 14, 988.

9. Z. Opial, Nonexpansive and monotone mappings in Banach spaces, Center for Dynamical Systems, Division of Applied Math., Brown University, Lecture Notes 67-1, 1967.

10. C. L. Outlaw, Mean value iteration of nonexpansive mappings in a Banach space, Pacific J. Math. 30 (1969), 747-750. MR 40 \#807.

11. W. V. Petryshyn, Construction of fixed points of demicompact mappings in Hilbert space, J. Math. Anal. Appl. 14 (1966), 276-284. MR 33 \#3147.

12. S. Reich, Some remarks concerning contraction mappings, Canad. Math. Bull. 14 (1971), 121-124. MR 45 \#1145.

Department of Mathematics, North Carolina State University, Raleigh, NORTH CAROLINA 27607 